\title{
Desigualdades y retos que enfrenta la educación en el Colegio de Ciencias y Humanidades durante la pandemia COVID 19
}

\author{
Verónica Marcela Espinoza-Islas \\ veronicamarcela.espinoza@cch.unam.mx \\ Colegio de Ciencias y Humanidades Vallejo, Universidad Nacional Autónoma de México, México. \\ https://orcid.org/0000-0003-3974-427X
}

\section{Fátima Sandra Rubiales-Sánchez}

fatima.rubiales@cch.unam.mx

Colegio de Ciencias y Humanidades Vallejo, Universidad Nacional Autónoma de México, México. https://orcid.org/0000-0003-3914-8590

\section{Ana Lilia Santana-Galindo}

analilia.santana@cch.unam.mx

Colegio de Ciencias y Humanidades Oriente, Universidad Nacional Autónoma de México, México. https://orcid.org/0000-0001-8174-7920

Recepción: 15 de julio del 2020 Aprobación: 08 de agosto del 2020 Publicación: 06 de junio de 2021

\section{Resumen}

Las prioridades educativas han sufrido grandes cambios a partir de la pandemia sanitaria derivada del Covid 19, evidenciado la enorme desigualdad socioeconómica y tecnológica, que limita aprovechar los sustitutos de la educación presencial y el aprendizaje posible.

Dentro de las problemáticas que nos encontramos es que no todas los alumnos cuentan con un equipo de cómputo como computadora de escritorio, laptop o tablet, y menos exclusivo para su uso personal, una gran mayoría tiene que compartir el equipo con familiares (Padres y hermanos) y no todas las escuelas tienen plataformas pertinentes para dar clases en línea o preparan a su personal docente al respecto, los alumnos no cuentan con hábitos de estudio o aprendizaje autónomo y autorregulado para realizar las actividades por sí mismos.

Por otra parte, esta migración de clases presenciales a clases en línea, expone los retos laborales desde el confinamiento. Cuando se trata de analizar el contexto de los profesores y estudiantes, obligados a adaptarse y cumplir sus funciones desde la distancia, es inevitable mencionar la brecha digital existente entre los actores del proceso de Enseñanza-Aprendizaje. 
El próximo reto es que la crisis del Covid-19 ponga sobre la mesa las exigencias de la Era Digital como una prioridad en la agenda educativa del país, sin olvidar la capacitación y el trabajo de los docentes, que en muchos casos va más allá de los límites de las aulas.

Palabras claves: Desigualdad, retos, tecnología, aprendizaje autónomo, recursos.

\title{
Inequalities and challenges facing education in the Colegio de Ciencias $y$ Humanidades during the COVID 19 pandemic
}

\begin{abstract}
Educational priorities have undergone great changes from the health pandemic derived from Covid 19, evidence of the enormous socioeconomic and technological inequality, which limits the use of substitutes for face-to-face education and possible learning.

Among the problems that we find is that not all students have a computer such as desktop computer, laptop or tablet, and less exclusively for their personal use, a large majority have to share the equipment with family members (Parents and siblings) and not all schools have relevant platforms to teach online or prepare their teaching staff in this regard, students do not have autonomous and self-regulated study or learning habits to carry out the activities on their own.
\end{abstract}

On the other hand, this migration from face-to-face classes to online classes exposes the work challenges from confinement. When it comes to analyzing the context of teachers and students, forced to adapt and fulfill their functions from a distance, it is inevitable to mention the existing digital gap between the actors in the Teaching-Learning process.

The next challenge is for the Covid-19 crisis to put the demands of the Digital Age on the table as a priority in the country's educational agenda, without forgetting the training and work of teachers, which in many cases goes beyond the limits of the classrooms.

Keywords: Inequality, challenges, technology, autonomous learning, resources. 


\section{Introducción}

La pandemia del COVID 19 ha dejado al descubierto desigualdades socioeconómicas en los alumnos del Colegio de Ciencias y Humanidades $(\mathrm{CCH})$, que traen consigo nuevos retos en la educación. Dejando entre ver que aunque la educación es un derecho humano y constitucional en México, no todos contamos con los mismos recursos para acceder a ella, pues, un ejemplo claro es que no todos los estudiantes cuentan con computadora de escritorio, laptop, tablet y/o acceso a internet en casa, lo cual genera diversas problemáticas que desglosamos en este trabajo de investigación.

Hablando de desigualdad de acuerdo con Marchesi en el 2000 "Se puede hablar de igualdad de oportunidades cuando todos los alumnos tienen formal y legalmente las mismas posibilidades educativas". Un nivel superior de igualdad se encuentra cuando, una vez garantizada la igualdad en el acceso, se proporciona un programa educativo similar a todos los alumnos y se evita, en consecuencia, que los que proceden de clases sociales populares están mayoritariamente representados en los programas menos valorados social y académicamente: aulas especiales para alumnos con discapacidades físicas, programas de educación especial para alumnos con capacidades diferentes, programas de garantía social incluyentes a los diversos estratos socioeconómicos (Marchesi, 1998 y Rivero, 1999). Ahora bien, la problemática que nos atañe no es en un espacio físico dentro de la escuela o el aula, es un problema de desigualdades derivado de la carencia de recursos económicos y tecnológicos en casa, que les permita hoy por hoy acceso a una educación incluyente

¿Cómo lograr que estas posibilidades se hagan accesibles a todos los alumnos, superando formas de acceso y la selección encubiertas?, en término más preciso ¿Cómo lograr la igualdad en el acceso a la educación?

Lo que nos lleva a ¿Cuales son ahora los retos que enfrenta la educación? Como nos hemos dado cuenta, actualmente, ya no es suficiente la manera en que se enseñaba y aprendía hace algunos años, pues la sociedad ha demostrado tener nuevas necesidades de: comunicación, conocimientos, habilidades, destrezas, estrategias de enseñanza y aprendizaje. Tiempo atrás, considerábamos analfabetas a aquellos que no sabían leer y escribir, sin

embargo, en la actualidad podríamos decir que existe otras categorías: los analfabetas

Verónica Marcela Espinoza Islas, Fátima Sandra Rubiales Sánchez y Ana Lilia Santana Galindo Desigualdades y retos que enfrenta la educación en el Colegio de Ciencias y Humanidades durante la pandemia COVID 19 
funcionales, los analfabetas gramaticales, los analfabetas por desuso y los analfabetas tecnológicos que son aquellos que no se adaptan a la nueva era de las TICS (Tecnologías de la Información y la Comunicación), TAC (Tecnologías del Aprendizaje y del Conocimiento) y TEP (Tecnologías para el Empoderamiento y la Participación).

La generación 2017-2020 enfrenta una gran ruptura a las estrategias de enseñanza y aprendizaje que se han manejado hasta hace unos meses, pues, han tenido que adaptarse a circunstancias fuera de lo común, como lo fue el sismos del 19 de septiembre de 2017 en la que se suspendieron labores en las instituciones por aproximadamente un mes y ahora la pandemia por la que estamos atravesando de SARS-COV2 llamado Covid-19, que llegó con la necesidad de adaptar las clases presenciales a clases en línea, apoyada de medios de comunicación, como lo fue la radio, televisión, uso de plataformas, etc., indiscutiblemente es una generación que se ha tenido que adaptar a estas circunstancias que han dejado huella en su aprendizaje.

En el presente trabajo nos interesa expresar lo que observamos con respecto a las problemáticas de desigualdad que enfrentaron nuestros alumnos en esta transición de las clases presenciales a clases virtuales durante la pandemia, dejando al descubierto que quizás tenemos el derecho a la educación pero existe una desigualdad latente en el acceso a los recursos tecnológicos que necesitamos en esta contingencia para acceder a ella y las carencias de hábitos de estudios que limitan el logro de los aprendizajes.

\section{Materiales y Métodos}

Para poder llevar a cabo esta investigación, se realizó una encuesta online la cual consta de 20 preguntas, aplicada a 200 alumnos del CCH Oriente y Vallejo. El objetivo de este trabajo es identificar los posibles factores que influyen en la desigualdad académica y los retos que enfrenta la educación en el Colegio de Ciencias y Humanidades.

El tipo de encuesta seleccionada fue mixta, las características de los preguntas

elegidas fueron: 1) Preguntas dirigidas, con el fin de estandarizar los resultados (13 preguntas) y 2) Preguntas abiertas para poder conocer la opinión de cada uno de los encuestados respecto a sus hábitos de estudio y condiciones escolares (7 preguntas). 
La encuesta se realizó a través de la herramienta Microsoft Forms ${ }^{\circledR}$, siendo de carácter anónimo por la privacidad de los encuestados.

\section{Resultados y Análisis}

Una de las primicias de esta investigación fue que los alumnos no tenían acceso a internet o alguno de los recursos tecnológicos (computadora, laptop o tablet) y por esta razón se les dificulto darle continuidad a sus cursos ordinarios, lo que coloca a este grupo de la población en una desigualdad académica.

Al preguntarles a los alumnos si tenían acceso a internet el $97 \%$ de ellos contestó que sí cuenta con dicho recurso y sólo el 3\% de ellos no, gráfico 1.

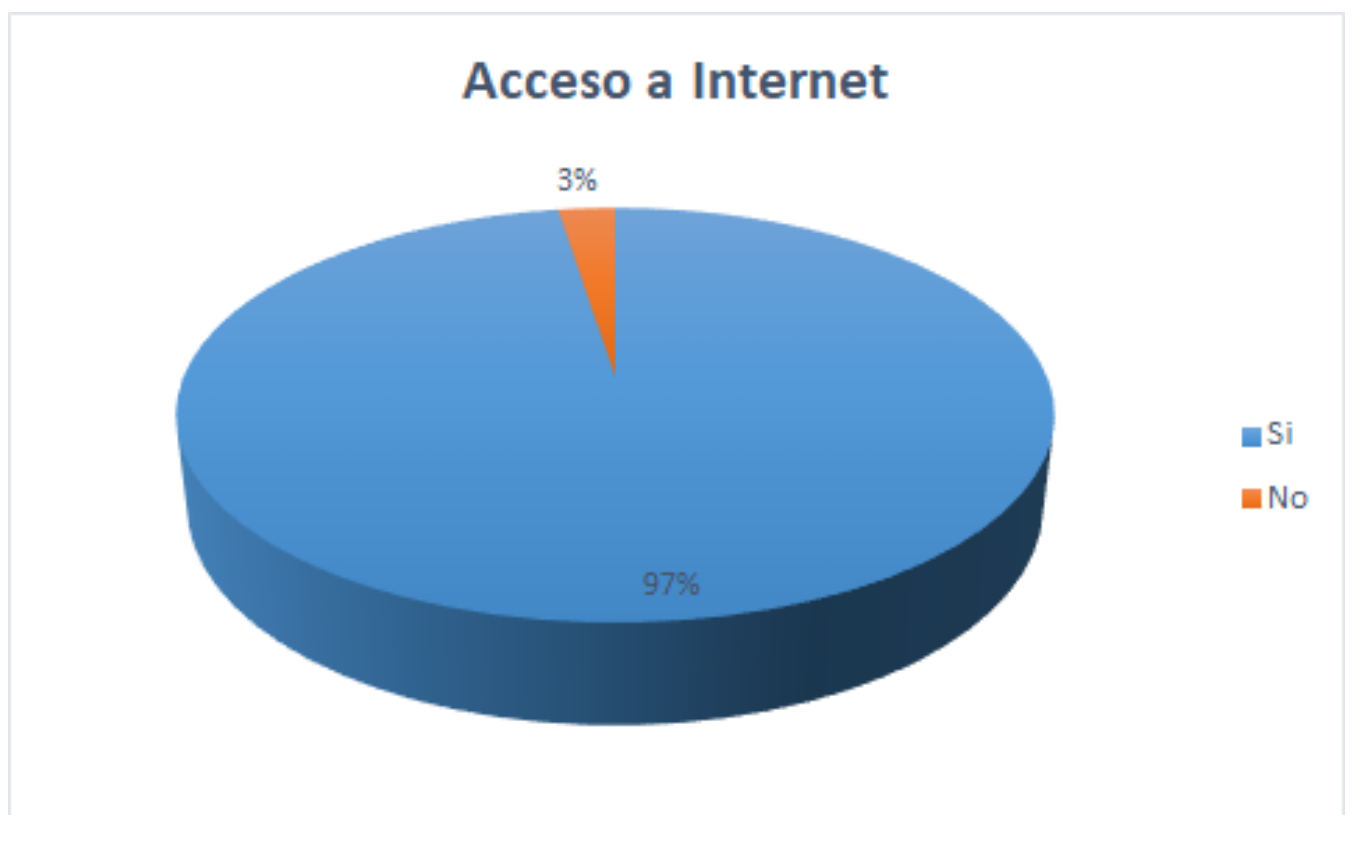

Gráfico 1. Cuentas con acceso a Internet

Cabe señalar que dentro del $97 \%$ menos del $2 \%$ mencionan que acababa de contratar el servicio en casa, por la necesidad que generó la pandemia de contar con él.

Consideramos importante saber si nuestros alumnos contaban con un dispositivo propio para hacer sus actividades académicas, es importante contemplarlo sobre todo si se dejan actividades programadas, ya que, esto les daría la misma posibilidad de elaboración y entrega, la respuesta a esta interrogante la encontramos en el gráfico 2 y 3.

Verónica Marcela Espinoza Islas,

Fátima Sandra Rubiales Sánchez y Ana Lilia Santana Galindo Desigualdades y retos que enfrenta la educación en el Colegio de Ciencias y Humanidades durante la pandemia COVID 19 
De esta forma, les preguntamos ¿Desde qué dispositivo te conectas?, pues, como docentes nos es útil reconocer cuáles son los recursos con lo que cuentan los alumnos para poder trabajar y acceder a las diversas plataformas educativas, elaborar textos, presentaciones, organizadores gráficos o cualquier otra evidencia de aprendizaje que conozcamos y favorecer el proceso de enseñanza y aprendizaje. Lo que encontramos fue que trabajan en los siguientes dispositivos: $15 \%$ computadora de escritorio, $34 \%$ laptop, $28 \%$ el celular y el $3 \%$ tablet, gráfico 2.

\section{Dispositivos utilizados}

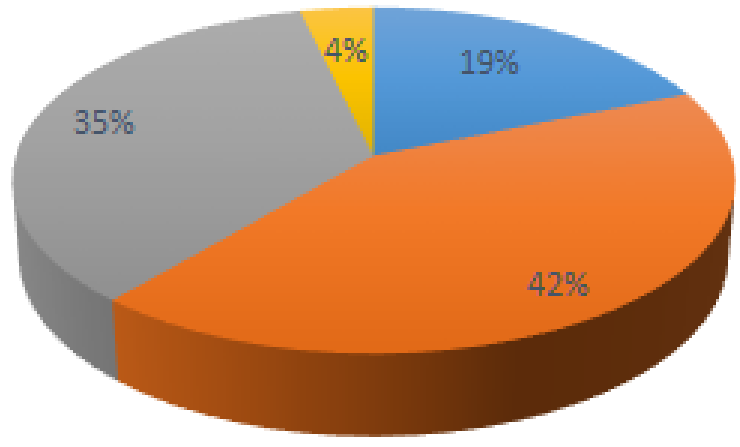

n Computador a de escritorio - Laptop

- Celular

Tablet

\section{Gráfico 2. Dispositivos desde los que trabajan los alumnos}

Como reflexión de esta pregunta, necesitamos considerar que una mayoría importante de nuestros alumnos debe trabajar desde su teléfono celular, la cual puede representar una problemáticas para los estudiantes, pues no todas las aplicaciones son compatibles con los dispositivos móviles. Por ejemplo, en el caso de matemáticas las fórmulas, no se visualizan desde el celular utilizando google forms, es necesario trabajarlo desde una computadora, sin embargo, al usar microsoft forms, utilizando "vista desde el celular" esta problemática no existe, en el caso de Moodle los exámenes que tienen la opción de arrastrar las respuestas no es posible hacerlo en el celular. 
Por un lado tenemos la problemática de contar o no con los recursos físicos, sin embargo, a pesar de tener un dispositivo en el cual pueden trabajar y acceder a internet encontramos que el 67\% de ellos debe compartir el dispositivo con algún familiar (Padres o hermanos), gráfico 3, lo que los pone en un turno de espera o conlleva a otra problemática, administrar las horas de trabajo.

\section{Dispositivos compartidos}

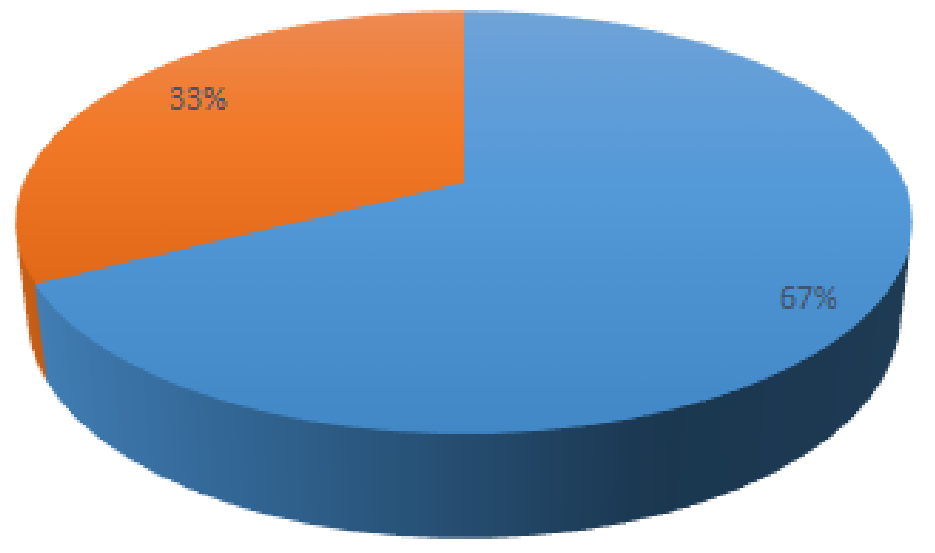

Gráfico 3. ¿Compartes tu dispositivo?

Por lo anterior podemos sugerir que como docentes debemos diseñar y programar las actividades con tiempo de antelación y no dejar actividades "sorpresa", asegurándonos que esta sean compatibles con los dispositivos móviles, quizás podamos generar una lista de actividades con fecha de entrega para que ellos las conozcan, al igual que la programación del examen con el tiempo de vigencia correspondiente, con la finalidad de que los alumnos puedan administrar sus tiempos en los dispositivos compartidos.

Tomando en cuenta que más del 50\% de los estudiantes comparten el dispositivo en el que trabajan, consideramos pertinente preguntarles ¿Por qué no se conectaron para terminar sus cursos ordinarios? ellos reportan que las actividades asignadas por el docente eran muchas y que no contaron con el tiempo suficiente para poder realizarlas pues su tiempo 
de conexión a internet y uso del dispositivo es breve, como se muestra en el gráfico 4, en el que la mayoría dispone de una a tres horas en promedio.

\section{Tiempo de uso del dispositivo}
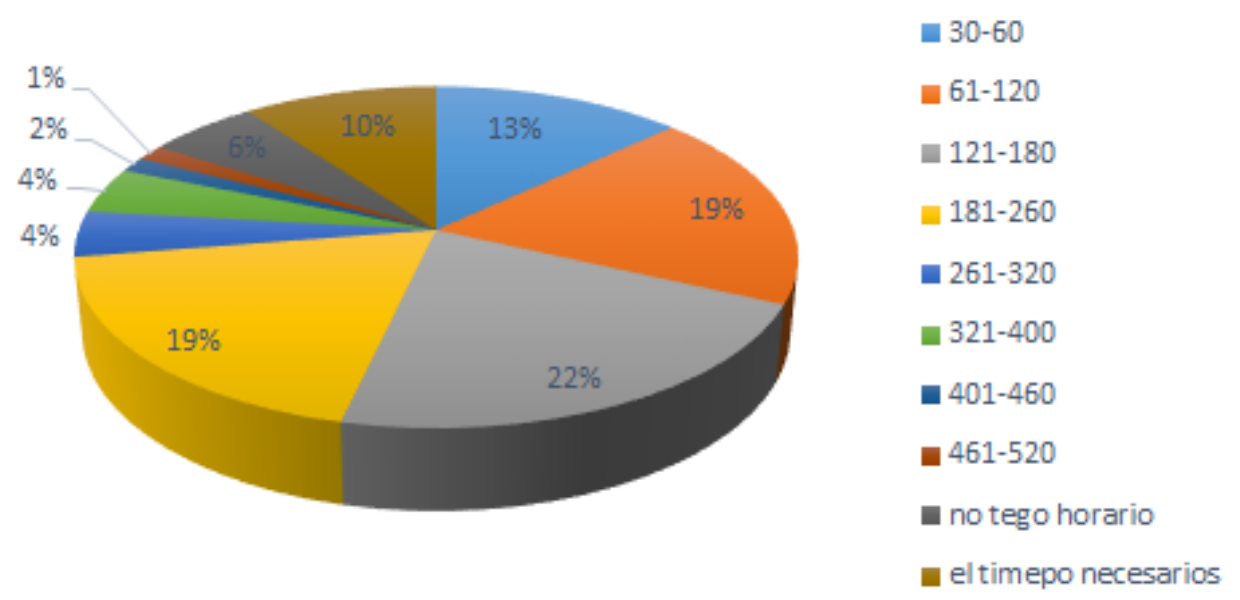

Gráfico 4. ¿Cuánto tiempo puedes utilizar tu dispositivo para realizar tus actividades académicas?

Por otra parte, cuando hablamos sobre algunas de las necesidades emergentes en la educación derivadas de esta pandemia pensamos la importancia de conocer cuales son los medios de comunicación que se establecieron para trabajar con los alumnos, lo que encontramos fue:

Como se muestra en el gráfico 5, antes de la pandemia el 45\% los alumnos no habían trabajado con alguna plataforma educativa paralela a las clases presenciales y el $55 \%$ si. Una interrogante que nos surge es ¿Cómo se usó esta plataforma previo a la pandemia? El principal objetivo que cumplen las plataformas digitales es facilitar la ejecución de tareas a través de programas o aplicaciones en un mismo lugar en la web (Giraldo, 2019), sin embargo nos surge la duda de como fue utilizada por el docente: quizás como medio de comunicación con el docente, como un repositorio de tareas o realmente fue usada para realizar trabajos complementarios de las actividades en clase, estos planteamientos nos surgen por que en una de las preguntas que viene posteriormente los chicos reportan tener dificultades con el uso de la plataforma, los que nos invita a reflexionar a profundidad sobre el tema. 


\section{Uso de plataforma antes de la pandemia}

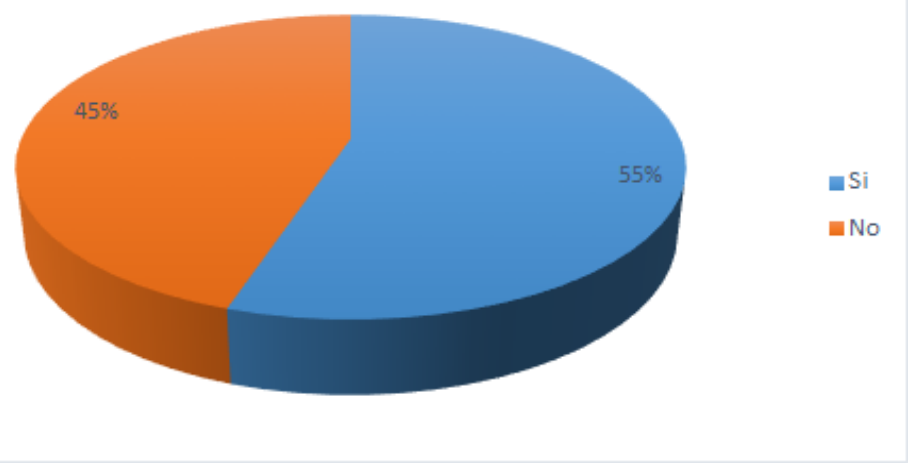

Gráfico 5. ¿Antes de la pandemia trabajabas alguna plataforma virtual en tus clases presenciales?

Es necesario mencionar que la Universidad Nacional Autónoma de México (UNAM) ha proporcionado a todos sus docentes diversas aulas virtuales previas a la pandemia, así como cursos y diplomados de capacitación para su uso, por esta razón consideramos pertinente preguntar a los alumnos ¿Qué plataforma educativa trabajaron? lo que observamos fue lo siguiente: el 36\% edmodo, 27\% Classroom, 23\% Aula virtual UNAM, 9\% Teams y $5 \%$ Moodle, es importante mencionar que todas estas plataformas son de acceso gratuito para la población estudiantil y docente, además de ser compatibles con dispositivos móviles (gráfico 6).

\section{Cuales son las plataformas que conoces}

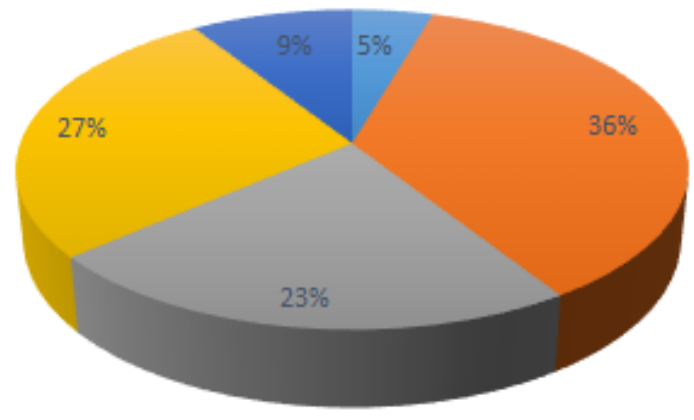

Moodle

Edmodo

Aulavirtual UNAM

- Classoom

- Teams

Gráfico 6. ¿En qué plataforma educativa trabajaste?

Verónica Marcela Espinoza Islas,

Fátima Sandra Rubiales Sánchez y Ana Lilia Santana Galindo Desigualdades y retos que enfrenta la educación en el Colegio de Ciencias y Humanidades durante la 
Consideramos que para hablar de los retos o futuros retos de la educación no solo necesitamos conocer los recursos con los que cuentan los estudiantes, sino también necesitamos saber si los alumnos presentaron alguna dificultad en esta transición de clases presenciales a clases en línea y ¿cuáles fueron esas dificultades que ellos reconocen en su calidad de estudiante? Lo que encontramos es que un $77 \%$ reconoce que tuvo dificultades para acoplarse a esta nueva modalidad en línea entre las causas que desglosan son: no sabían usar la plataforma, las instrucciones del docente no eran claras, la falta de recursos pues algunos no contaban con internet o con un dispositivo propio para trabajar y debían administrar sus tiempos con quien lo comparten (gráfico 7).

\section{Dificultades durante el trabajo en línea}
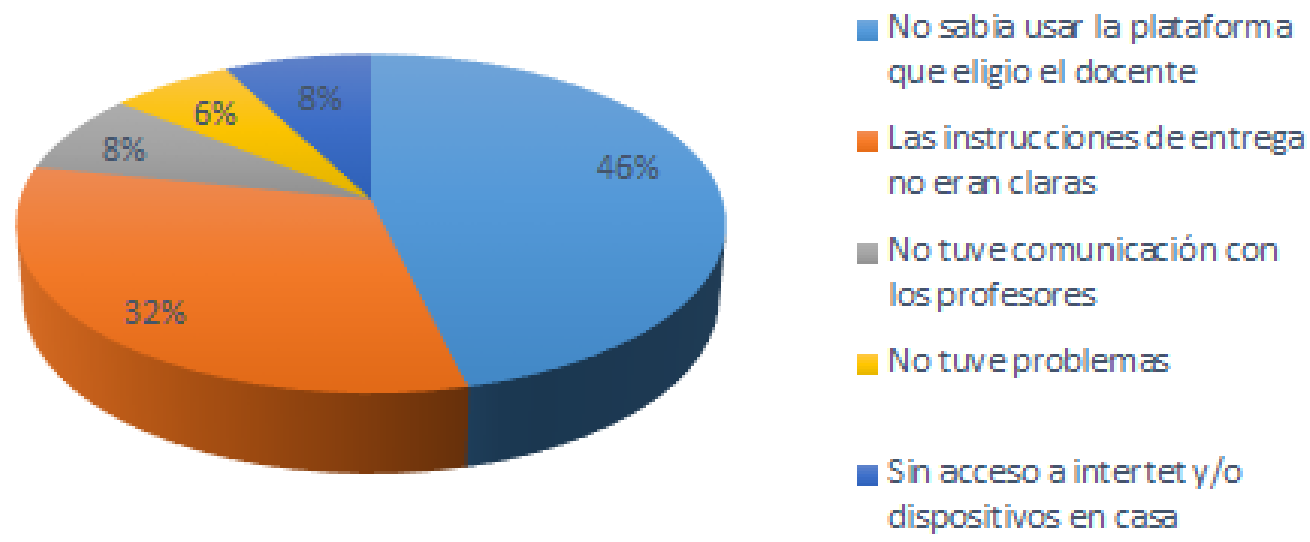

\section{Gráfico 7. ¿Qué dificultades presentaste para trabajar en línea?}

Es importante destacar que los estudiantes reconocen que tienen la necesidad de que los temas les sean explicados, pues, las actividades que les fueron asignadas no les fueron suficientes para comprender los temas $42 \%$, así mismo, el $31 \%$ consideran que las actividades que les fueron asignadas no eran para hacerse en línea sino para el aula, es decir, deben trabajarse de manera presencial, con la guia del docente para explicarles el procedimiento o aclarar dudas del proceso, un $17 \%$ considera que necesita tener un tiempo definido para realizar sus actividades en casa, lo que nos refiere a los hábitos de estudio y el $12 \%$ restante 
reporta dificultades por las obligaciones familiares que no tenían antes de la pandemia, como el cuidado de los hermanos, abuelos o familiares enfermos (gráfico 8).

El reto de esta problemática es cómo hará el alumno para administrar su tiempo para el desarrollo de las actividades en las diversas asignaturas que cursará y si el docente está consciente de que dichas actividades deben apegarse a el tiempo destinado para su clase.

\section{Necesidades académicas en la transición}
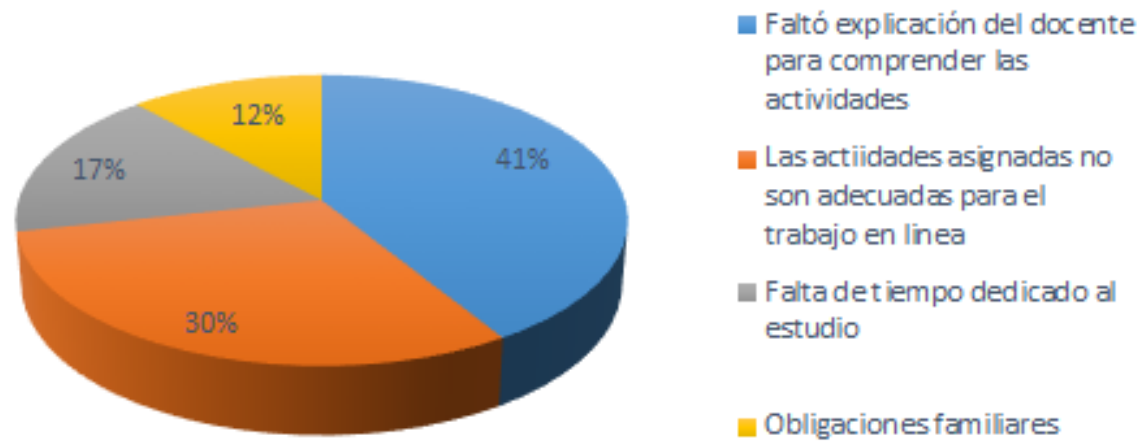

Gráfico 8. Necesidades académicas detectadas por los estudiantes derivadas del trabajo en línea

Con respecto a la problemática de los tiempos para estudiar o hacer actividades en casa, un reto significativo para la docencia ahora en línea, son los hábitos de estudio del alumnos, pues se requiere de alumnos autónomos en su aprendizaje y autorregulados para el desarrollo de las actividades en casa, por lo que les preguntamos ¿Qué estrategias usas para estudiar y no reprobar? lo que encontramos es que la mayoría de los estudiantes realizan diversas actividades pero en clase, pues no realiza investigación aparte o lee las actividades complementarias como lecturas, investigación de conceptos, guías, etc., que deja el docente fuera del aula el, $50 \%$ dice que si lo hace, el $45.8 \%$ menciona que a veces y el $4.2 \%$ dice que no, así mismo, les preguntamos si pasan los apuntes en limpio o hacen esquemas complementarios a lo visto en clase, el $20.8 \%$ dice que si, el 45,8\% dice que a veces y el $33.3 \%$ dice que no. 


\section{Actividades complementarias de clase}

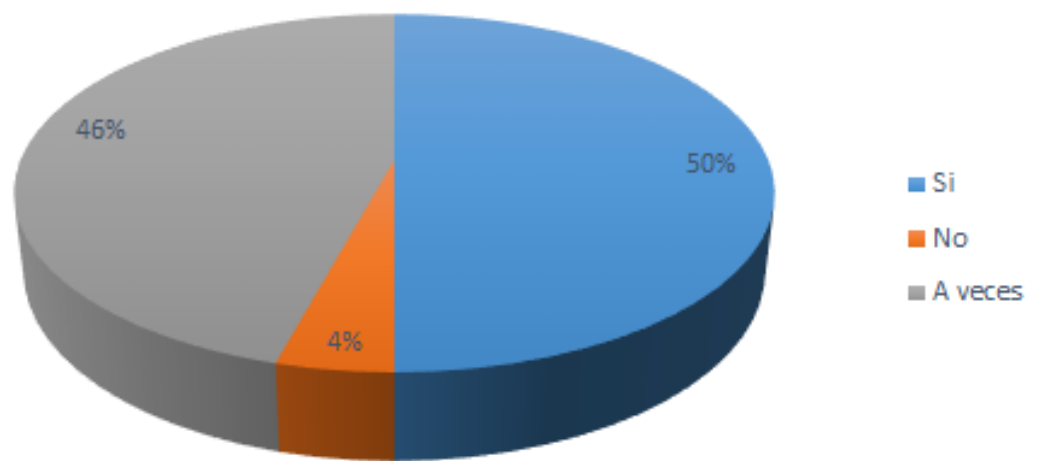

Gráfico 9, Actividades complementarias de clase

Por lo anterior, podemos decir que sus hábitos de estudios se reducen a realizar actividades dentro del aula como: entregar trabajos $25 \%$, asistir a clase y participar en ella $25 \%$, poner atención $20.8 \%$, estudiar de los apuntes $16.7 \%$, estudiar de otras fuentes $8.3 \%$ y exponer dudas $4.2 \%$ (gráfico 10), evidentemente la carencia del aprendizaje autónomo limita el desarrollo cognitivo del alumno para el logro de los aprendizajes.

\section{Que estrategia usas para no reprobar}
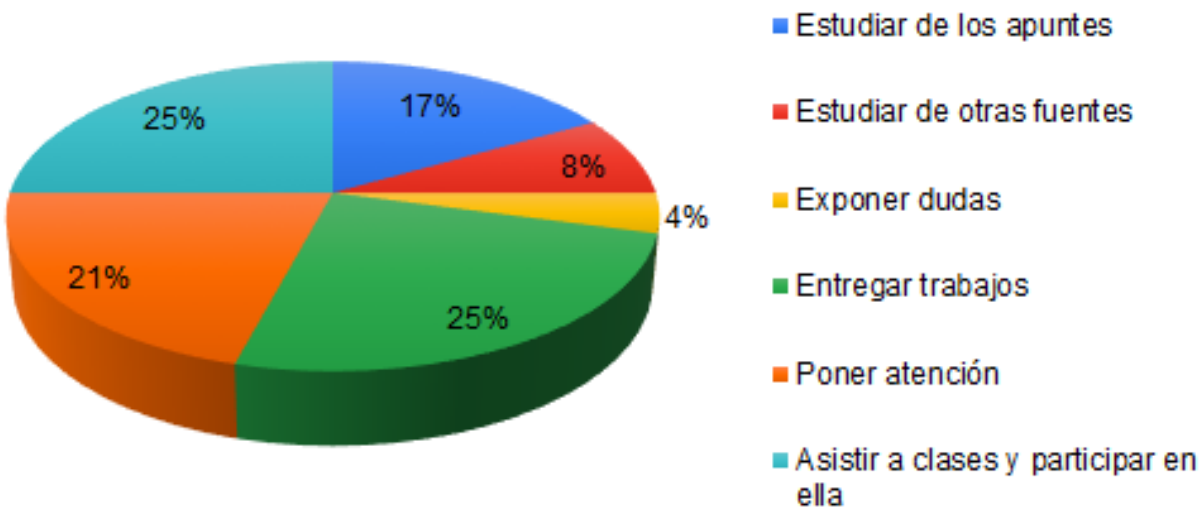

Gráfico 10. ¿Qué estrategias usas para no reprobar? 


\section{Conclusiones}

En la actualidad la sociedad demanda individuos creativos, emprendedores, críticos, competentes en las TIC, TAC y TEP, autónomos, con alta capacidad comunicativa y tolerantes a la opiniones diversas de pensamiento, que se adapten fácilmente a los ambientes laborales, capaces de trabajar con cualquier persona, en cualquier lugar y momento. Los alumnos de hoy no sabemos qué serán en el futuro, pero deberán tener los recursos necesarios para adaptarse a las nuevas necesidades.

A pesar de los esfuerzos realizados por diversas instituciones educativas para dar continuidad a los aprendizajes, como es el caso de la UNAM, tanto alumnos como docentes nos encontramos atravesando una etapa de cambio, en la que no solo basta llevar a cabo una actualización docente sino que se requiere de una actualización continua de los actores involucrados en el proceso de enseñanza aprendizaje, recursos físicos como laptop, tabletas, computadoras personales, etc., y el acceso a Internet que se ha vuelto fundamental para llevar a cabo el proceso educativo.

Dentro de los retos de la educación en esta transición de clases presenciales a clases en línea podemos preguntarnos ¿Qué ventajas encuentran los estudiantes al trabajo en línea? algunas de las respuestas que encontramos fueron:

"ser autodidacta, recibir apoyo en casa en caso de que se requiera, acceso a internet para realizar investigaciones, ahorrar el tiempo de desplazamiento del plantel a sus casas y viceversa, ya que algunos de los estudiantes dedican de 1:30 a 2 horas para llegar a la escuela, en casas se trabaja con mayor comodidad, el tiempo de entrega de las actividades es más accesible, es más fácil investigar, no gasto en pasajes”.

Es importante analizar las dos caras de la moneda, por lo que indagamos en, ¿Qué desventajas encuentran los alumnos al trabajo en línea? :

"los maestros no se comunican y no te explican, las fallas técnicas como la falta del internet, la computadora, etc., muchos no podemos entender las cosas solo leyendo necesitamos que nos explique físicamente, fallas de los medios virtuales (videollamadas, plataformas, etc.), no hay contacto con los maestros, en la exposición de dudas el maestro tarda en contestar y

Verónica Marcela Espinoza Islas,

Fátima Sandra Rubiales Sánchez y Ana Lilia Santana Galindo Desigualdades y retos que enfrenta la educación en el Colegio de Ciencias y Humanidades durante la 
eso retrasa el trabajo, el trabajo en equipo se complica, no hay horas específicas de trabajo, me da flojera hacer las actividades, el exceso de trabajo"

Como podemos observar dentro de las ventajas que los alumnos encuentran está la autonomía del aprendizaje y poder realizar las actividades en sus horarios, sin embargo, consideramos importante reconocer la inconmensurabilidad que existe en ello pues la gran mayoría de los alumnos carecen de hábitos de estudios y de una disciplina para el estudio autorregulado y autodidacta, como docentes podríamos reflexionar si este pensamiento del alumno es utópico o si se reflejo en la calidad de los trabajos entregados y por supuesto en el logro de los aprendizajes, lo cual nos deja un tema muy interesante sobre la mesa de debate y futuras investigaciones.

Por otra parte como ellos mismos mencionan en las desventajas les da flojera realizar los trabajos, no tienen horas específicas para trabajar y necesitan del acompañamiento del docente para el logro de los aprendizajes, es bien sabido que en el proceso de aprendizaje tenemos muchas formas de aprender y apropiarnos del conocimiento sin embargo es inherente que nuestros alumnos necesitan del docente y que la comunicación asincrónica que se establece en las plataformas o redes sociales no les es suficiente, pues, requieren del contacto físico o interacción cara a cara.

Dentro de los retos que enfrenta la educación en este momento de transición es establecer un proceso de comunicación claro y afectivo, mediante una comunicación asincrónica que debe subsanarse, la organización de las actividades y aprender como docentes que el tiempo es un recurso y debemos apegarnos a horarios establecidos ya que la gran mayoría de nuestros estudiantes carecen de hábitos de estudios. Por otra parte, contemplar que la tecnología también es impredecible y tiene fallas, por lo que como docentes debemos de prever estos detalles y por último pero no menos importante reconocer que el docente está en continua construcción y capacitación de nuevas herramientas tecnológicas y didácticas, que lo apoyen en su labor del día a día.

Es una realidad evidente que nos encontramos en una etapa de cambio, en la que sin duda alguna debemos adquirir nuevos conocimientos tecnológicos, didácticos y pedagógicos, que nos permitan adaptar nuestras clases presenciales a un sistema de clases en línea, que ayude a los alumnos a adquirir los conocimientos y aprendizajes necesarios correspondientes 
al programa de estudios. Quizás a primera vista, esta sea una situación temporal, sin embargo, las herramientas que se adquieran en este periodo de aprendizaje, lleguen para quedarse y sean una herramienta más, para fortalecer y enriquecer nuestra labor docente.

\section{Referencias}

Fernández Gómez, E. (2009). U-learning: el futuro está aquí . México D.F: Editorial Alfaomega.

Giraldo, V. (2019). Las plataformas digitales son soluciones online que posibilitan la ejecución de diversas tareas en un mismo lugar a través de internet. recuperado el 28 de julio 2020 de: https://rockcontent.com/es/blog/plataformasdigitales/\#: :text=El\%20principal\%20objetivo\%20que\%20cumplen,la\%20necesida d\%20de\%201os\%20usuarios.

Jiménez E. y Bosada M. (2019). Los 6 retos de la educación para el nuevo curso escolar. Educaweb, recuperado el 16 de julio 2020 de https://www.educaweb.com/noticia/2019/09/12/6-retos-educacion-nuevo-curso$\underline{\text { escolar-18902/ }}$

Marchesi, A. (2000). Controversias en la educación española. Madrid, Alianza.

Marchesi, A. y Martín, E. (1998). Calidad de la enseñanza en tiempo de cambio. Madrid, Alianza. 
Nuttall, D.L. (1992). «The functions and limitations of international education indicators». En: The OECD, 1992, International Education Indicators. París, OCDE.

OCDE. (1998). Education at a glance. Indicators 1998. París.

PNUD. (1999). Informe sobre el desarrollo humano. Madrid, Mundi Prensa.

Prensky, M. (2010). From Digital Natives to Digital Wisdom: Hopeful Essays for 21st Century Learning. Newbury Park: Editorial Corwin.

Rivero, J. (1999). Educación y exclusión en América Latina. Reformas en tiempos de globalización. Buenos Aires, Niño y Dávila. 